Research Article

\title{
Internet of Things (IoT) Technology for the Development of Intelligent Decision Support Education Platform
}

\author{
Jinhua Liu $(\mathbb{D}$, Caiping Wang, and Xianchun Xiao \\ Jiangsu Vocational College of Agriculture and Forestry, Jurong 212400, China \\ Correspondence should be addressed to Jinhua Liu; liujinhua@jsafc.edu.cn
}

Received 24 October 2021; Accepted 2 December 2021; Published 22 December 2021

Academic Editor: Rahman Ali

Copyright ( $) 2021$ Jinhua Liu et al. This is an open access article distributed under the Creative Commons Attribution License, which permits unrestricted use, distribution, and reproduction in any medium, provided the original work is properly cited.

Improving the intelligence of teaching environment and making the multimedia teaching equipment has become a major concern of the colleges and universities. To this end, the design of Internet of Things (IoT) technology based wisdom of higher education platform is of great interest. Designing the structure of online management platform for college education and realizing the functions of examination result inquiry, online teaching, and attendance management have gained more importance in the educational research. The wisdom classroom is the key structure of the wisdom education platform. A smart classroom architecture based on IoT technology is designed, which connects with traditional network facilities through the IoT gateway. Different layers of the architectures have been designed and implemented. The proposed platform tests results and shows that the intelligent education platform can effectively control classroom utilization and has high throughput, low application latency, and good practicability.

\section{Introduction}

With the rapid development of technology, traditional teaching methods cannot meet the needs of modern teaching technology, and some great limitations also appeared in multimedia classroom: learners cannot participate in the learning process, demonstrating that teaching activities easily lead to cognitive overload [1]. Therefore, it is urgent to create a new classroom environment that is suitable for individual learning and interactive teaching. The mobile Internet, cloud computing, big data, Internet of Things, and other advanced technologies and equipment are made full use of to improve the learning environment and build an intelligent teaching system, which has become the embodiment of this learning trend at present [2]. At present, the existence of a large number of educational resources has led to serious problems of scattered resources and low-level redundant construction in the construction of education application platforms. Although education informatization has made certain achievements in network environment and hardware construction, it has not yet achieved intelligent services under big data, resulting in the unbalanced development of the construction of education platforms and the inability to provide one-stop online services for teachers, administrators, and students, which has become an important difficulty in affecting education informatization. Intelligent education is to apply the concept of informationization in the field of education, comprehensively and deeply apply modern information technology, and accelerate the process of education reform and development [3].

In [4], based on the existing theoretical and practical achievements of smart classroom, this paper puts forward the definition and connotation of the new generation of smart classroom and constructs the smart classroom model in the smart era from the aspects of system service concept, system architecture, platform construction, and application mode, so as to promote the innovation of subject smart teaching and truly realize personalized learning and individualized teaching and promote the development of learners' knowledge into wisdom. This paper expounds the overall architecture of "cloud platform end" of intelligent classroom intelligent service platform, aiming to open up the data transmission and communication channels of intelligent cloud service, classroom intelligent platform, and 
intelligent terminal, provide resource services, interactive services, and teaching tools, and build an integrated and intelligent learning environment based on intelligent information technology, which lays a foundation for the practical application of intelligent teaching in the future. In [5], we investigate and analyze the mining technology of two kinds of educational big data: teaching behavior and massive knowledge resources. Secondly, four key technologies in teaching links are focused on, guidance, recommendation, Q\&A, and evaluation, including learning path generation and navigation, learner portrait and personalized recommendation, intelligent online Q\&A, and fine evaluation, and then compares and analyzes the mainstream intellectual education platforms at home and abroad. Finally, the limitations of the current research on intelligence education are discussed, and the research and development directions of intelligence education, such as online intelligent learning assistant, learner intelligence assessment, networked group cognition, and causality discovery, are summarized. In [6], in order to realize the one-stop online service in the process of education, an intelligent education platform including data resource database, big data analysis layer, intelligent education information cloud service layer, application service layer, and presentation layer is designed based on big data analysis technology. The big data analysis layer uses the HBase database combined with the SQL computing execution engine to analyze the smart education data and transmits the analyzed student, teacher, and resource information to the smart education information cloud service layer. The intelligent education information cloud service layer enables platform users to enjoy platform storage files, course management, course publishing, and other services through user authentication, service binding, and service provision. The administrator of the online learning module in the intelligent education information cloud service layer reviews teachers' lesson preparation materials and students' learning resources, teachers' online or video teaching, and the realization of students' course selection and determination functions, so as to realize the online information exchange between teachers, administrators, and students.

IoT protocols are divided into different types based on the role they play within the network. Among many others, there are protocols used in connectivity infrastructure (e.g., 6LowPAN), communications (Wi-Fi, Bluetooth), data transmission (MQTT, CoAP, XMPP), security (DTLS), and device management as well as telemetry [7].

However, the focus of the above literature research is on the integration and digitization of multimedia teaching, ignoring the control of classroom modules and resulting in the overall application of intelligent teaching platform being not ideal. Therefore, the design of intelligence education platform based on Internet of Things technology is proposed.

Key contributions of the research include the following:

(i) Design of Internet of Things (IoT) technology based platform for higher education.

(ii) Designing the structure of online management platform for college education, realizing the functions of examination result inquiry, online teaching, and attendance management, which have gained more importance during the educational research.

(iii) Designing a smart classroom architecture based on IoT technology, which connects with traditional network facilities through the IoT gateway.

\section{Design of University Intelligent Education Platform Based on Internet of Things Technology}

2.1. Internet of Things Technology. Internet of Things is an interconnected network that uses various information sensing devices such as radio frequency identification devices, infrared sensors, laser scanning, and GPS and collects real-time information that requires monitoring, connection, interaction, and so forth. The core technology of Internet of Things is to realize the whole process of information acquisition, transmission, storage, processing, and application. The real-time interactive perception of information among "human-human," "human-object," and "material-object" is realized, and the Internet of Things can connect the real world and virtual world as well as the items in the real world, effectively supporting human-computer interaction, personal-human interaction, and human-human social interaction. Internet of Things can be divided into three layers: perception layer, network layer, and application layer. Sensing layer is the infrastructure layer of the Internet of Things, responsible for collecting all kinds of data information of classroom environment equipment and consisting of various sensors and sensor gateways, and it is the source of the Internet of Things information [8].

The network layer utilizes communication networking technology, including wired technology and wireless technology, such as Ethernet, Wi-Fi wireless network, cellular mobile network, and ZigBee technology, to connect various hardware devices in the classroom to form an interconnected network platform; meanwhile, the processing layer obtains data information and stores, fuses, and remotely transmits the data information. The application layer is the processing layer. By mining and processing the data information of the perception layer, the application platform of intelligent teaching service, such as indoor environment monitoring platform, hardware control platform, and intelligent teaching management platform, is constructed [9].

\subsection{Connection between Internet of Things Technology and} Intelligent Education Platform in Colleges and Universities. There are a series of problems in traditional campus and teaching platform, such as difficult to realize intelligent management and control, high energy consumption and lack of uniform application analysis, abnormal environment and lack of security warning, and difficult to exchange information in complex scenes. With the help of Internet of Things technology, daily teaching management and various teaching data can be automatically pooled to explore the relationship between the data. In the learning space based on 
the Internet of Things, it can effectively realize the combination of physical space and digital technology, improve the relationship between learners and the learning environment, strengthen the communication, collaboration, and sharing among learners, and effectively promote open learning and personalized learning. Internet of Things technology can provide the software and hardware equipment and technology needed for the operation of the intelligent teaching platform, support flexible teaching and learning methods, support the accumulation of rich learning experience, and provide strong technical support for precision teaching activities [10]. Intelligent teaching platform not only emphasizes the advancement of equipment and technology but also emphasizes the flexible application of technology to support the learning process and strengthen the learning effect.

\subsubsection{Information Processing in Intelligent Teaching Process.} What the intelligent teaching process information processing platform should solve is how to make the intelligent teaching platform process a lot of data information more independently, reduce people's participation, and improve the efficiency of information processing. The connection of teaching software and hardware cannot produce value. Through the Internet of Things, we can combine a great deal of information and data generated in the teaching process with intelligent analysis, analyze, process, and utilize the data and information, and explore the value of the information therein. This is the meaning of intelligent interconnection. The information processing of intelligent teaching process is composed of two parts, front-end intelligent information processing and back-end intelligent information analysis, and integrates with IoT.

Information analysis and processing can be divided into two aspects: one is to use the equipment based on the Internet of Things technology to automatically recognize the learner's individual, voice, character, and so forth and its general process includes the collection of sample data, digitized processing of information, preprocessing, extraction of data characteristics, comparison with the preset standard mode, classification recognition, and so forth [11].

The other is the understanding of natural language. Understanding natural language and the generation of natural language is the key to intelligent processing of information in intelligent teaching platform. Understanding human natural language is mainly based on intelligent processing and analysis of information.

\subsubsection{Information Exchange and Coordination of Intelligent} Teaching Platform. The information exchange and coordination platform based on Internet of Things technology is to use modern advanced technology to change the traditional teaching mode of "oral paper" as the medium. Under the digital environment, the information transmission and mutual coordination among various devices can significantly improve the teaching efficiency. End-to-end automation and human-computer collaboration can simplify routine teaching tasks and improve learners' ability to create and solve problems individually [12]. The advantage of endto-end transmission is that the transmission delay is small, which can greatly save time in the process of teaching information exchange. Man-machine cooperation technology can provide new ways and means for teaching activities, provide an optimal learning path for learners, and seek a more suitable individual education mode.

At the same time, intelligent communication and coordination can liberate teachers from the repetitive and mechanical work and focus on teaching work and humancomputer cooperation to complete previously impossible intelligent work.

\subsubsection{Information Feedback of Intelligent Teaching Process.} Intelligent teaching process information feedback platform can affect the result of teaching object's input information and the output of data information again. There are two aspects in its realization: one is the visualization of process state information and the other is the instantaneous presentation of feedback information. It is an inexorable trend to integrate information visualization technology and instant information feedback technology into the classroom. Teaching process information visualization refers to the process in which people use the natural ability of quick recognition to transform data information and knowledge into visual form, so as to realize the comprehensive analysis and mastery of teaching state and to optimize the production of teaching results. Using modern visualization technology to reveal the laws that traditional teaching methods cannot show, multidimensional abstract data will be displayed as two-dimensional or three-dimensional graphics to achieve deep interaction between people and information data for timely feedback of teaching information [13]. The intelligent teaching platform can capture the learner's learning behavior information in real time through various sensor devices connected by the Internet of Things and collect the statistical data and give immediate feedback after the platform analysis and processing. This real-time feedback platform is helpful for students to participate in the interaction, accurately grasp the learners' learning, and facilitate teachers to adjust teaching activities and optimize the teaching process.

\subsection{Structure of Online Management Platform for Higher} Education. The online management platform of college education is a platform to improve the management efficiency and quality of college education and make college education management informationalized and standardized. The overall structure diagram of the online management platform for college education based on Internet of Things technology is shown in Figure 1.

The online management of college education is realized through the online management platform of college education based on Internet of Things technology, which includes application service layer, education resources layer, and infrastructure layer.

The infrastructure layer of the platform integrates the storage devices, network facilities, computers, servers, and 


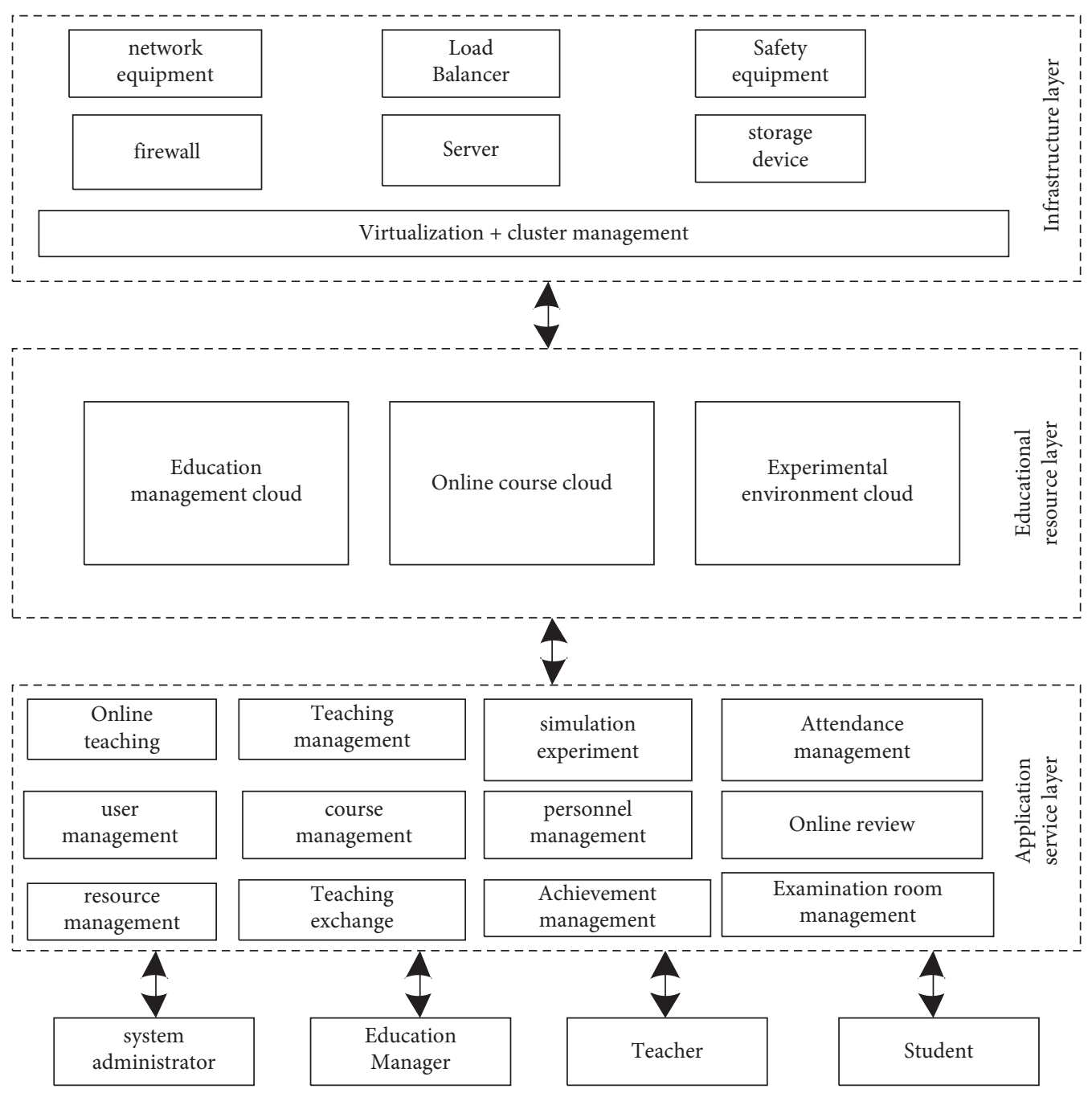

FIGURE 1: Overall structure of the platform.

other resources of the platform, integrates all its facilities into a stable unified IOT technical infrastructure environment by virtue of virtual technologies of software and hardware, and provides equipment resources for the platform.

The educational resources layer includes cloud resources of experimental environment, cloud resources of education management, and cloud resources of network courses, and, through the educational resources layer, the classification, screening, collection, and other standardized management operations of massive college education data are realized by using the data clustering algorithm of cloud computing, and the scattered massive education data are unified by using the educational resources layer to provide cloud services for the online management of college education $[14,15]$.

The application service layer of the platform includes attendance management, simulation experiment, online teaching, attendance management, score management, and many other applications.

After the IoT technology of the platform is deployed by using the method of the IoT Technology Department, users such as students and teachers send service requests through the application service layer, and the platform sends user requests to the education resources layer, and the education resources are mined by using the data clustering algorithm of cloud computing, and the resources required by users are sent to the user display interface after data mining [16].

\subsection{Smart Classroom Architecture Based on Internet of Things} Technology. Based on the application architecture of Internet of Things, there are three layers: perception layer, transport layer, and application layer. The perceptual layer is deployed in each multimedia classroom, and various IoT networking technologies can be used to connect to various traditional network facilities through IoT gateways. The communication protocol of the gateway can be lightweight, and the complex application layer processing function cannot be deployed, so the gateway can be decoupled from the backstage application business logic. The application layer processes the collected sensing data, such as the data based on the brightness sensor, to determine if it is in class time and the classroom brightness is below the threshold; then it issues an order to turn on the light $[17,18]$. 
The instruction is transmitted to the environmental equipment controller of the designated classroom (the controller may, according to different instruction types, respectively, control the opening, regulation, and other operations of lighting, air-conditioning, air purifier, and other equipment), and the environmental equipment controller receiving the instruction inquires about the address of lighting equipment of the classroom and sends instructions through the RXTX module to the appropriate port so as to realize the control of the equipment, that is, turn on the lighting. The process of controlling multimedia instructional equipment is similar and needs not be repeated. The architecture of the wisdom classroom platform presented in this paper is shown in Figure 2.

The administrator can display the comprehensive information of the platform on the big screen of the monitoring center, and the teacher can query the related information of the class through the client program on the computer of the multimedia classroom. The smart classroom server is used for deploying classroom management platform (application server) and device access service middleware (DAS) and docking with campus card information interface and course scheduling platform and interconnecting and sharing information. Administrators or teachers through the download of the relevant App can always monitor the platform integrated information or query to the designated class related information.

\subsection{Function Module of Online Management Platform for} Higher Education. According to the management requirements of college education online management platform, the functional structure of college education online management platform based on Internet of Things technology is designed, as shown in Figure 3.

The functional structure of the platform includes five parts: information management module, achievement management module, attendance management module, teaching and resource management module, and other management modules.

The information management module has all the information management functions of students, teachers, platform administrators, and education administrators. Students, teachers, and education administrators can only add, modify, delete, and query their own information related to the platform administrator that has all user information management authority. The teacher has the function of updating, deleting, adding, and inquiring the students' achievements. The students can only inquire their own achievements through this function module.

Attendance management module has the function of recording students' class, homework, and exam attendance information. Teachers evaluate students' course performance through attendance management module.

Teaching and resource management module includes online examination, online teaching, downloading of teaching resources, and teaching trends. Students can take examinations and study online through this module. Teachers can publish the teaching plan and teaching trends to the part of teaching trends. Students and teachers can download teaching resources through the part of teaching resources.

Other management modules include simulation experiment, personnel management, examination room management, and other functions to meet the diversified management needs of the platform.

\subsection{Design of Intelligent Education Information Cloud Service} Layer. The cloud service layer of intelligent education information connects platform users with platform application services, which is the core level of the intelligent education platform, specifically including two modules: online learning and platform information release and management. The online learning module includes teaching, review, and exchange functions. The release and management of platform information realize platform information management through user registration accounts, authority allocation, and identity review. The relationship between platform users and platform services in the cloud service layer of intelligent education information is shown in Figure 4.

Intelligent education information cloud service center includes user and service. Users shall complete the registration and login of the intelligent education platform through the platform registration, user management, and login verification operation and manage and master all the basic information of all registered teachers, administrators, and students of the intelligent education platform, so as to facilitate the centralized management of the intelligent education platform; in terms of services, the intelligent education platform services shall authorize the platform services to the platform users through service registration, service search, and service management, and the intelligent education platform services shall be authenticated by users, service binding, and service provision, so as to realize the user authorization of the platform storage files, course management and course release, and other services, and users may enjoy the intelligent education platform services.

\subsection{Implementation of Platform}

2.7.1. Internet of Things Technology Deployment. In order to enhance the adaptability of the platform to user visits, connect the platform browsing server with the private cloud of educational resources layer, and avoid resource waste when concurrent users are too low by reducing virtual resources and avoid insufficient access to resources when concurrent users are too high by enhancing virtual resources, the proposal of the IoT Technology Department is shown in Figure 5. Two load balancers enable the platform to meet the access requirements of too many users, achieve load balancing, and distribute the traffic to the platform by using the minimum connection number first policy. The virtual resources of the IoT technology shall be dynamically adjusted by using the monitoring and scheduling server, and the monitoring and scheduling server may collect the relevant operation parameters of the virtual machine, database, and server. The IoT technology shall utilize the collected 


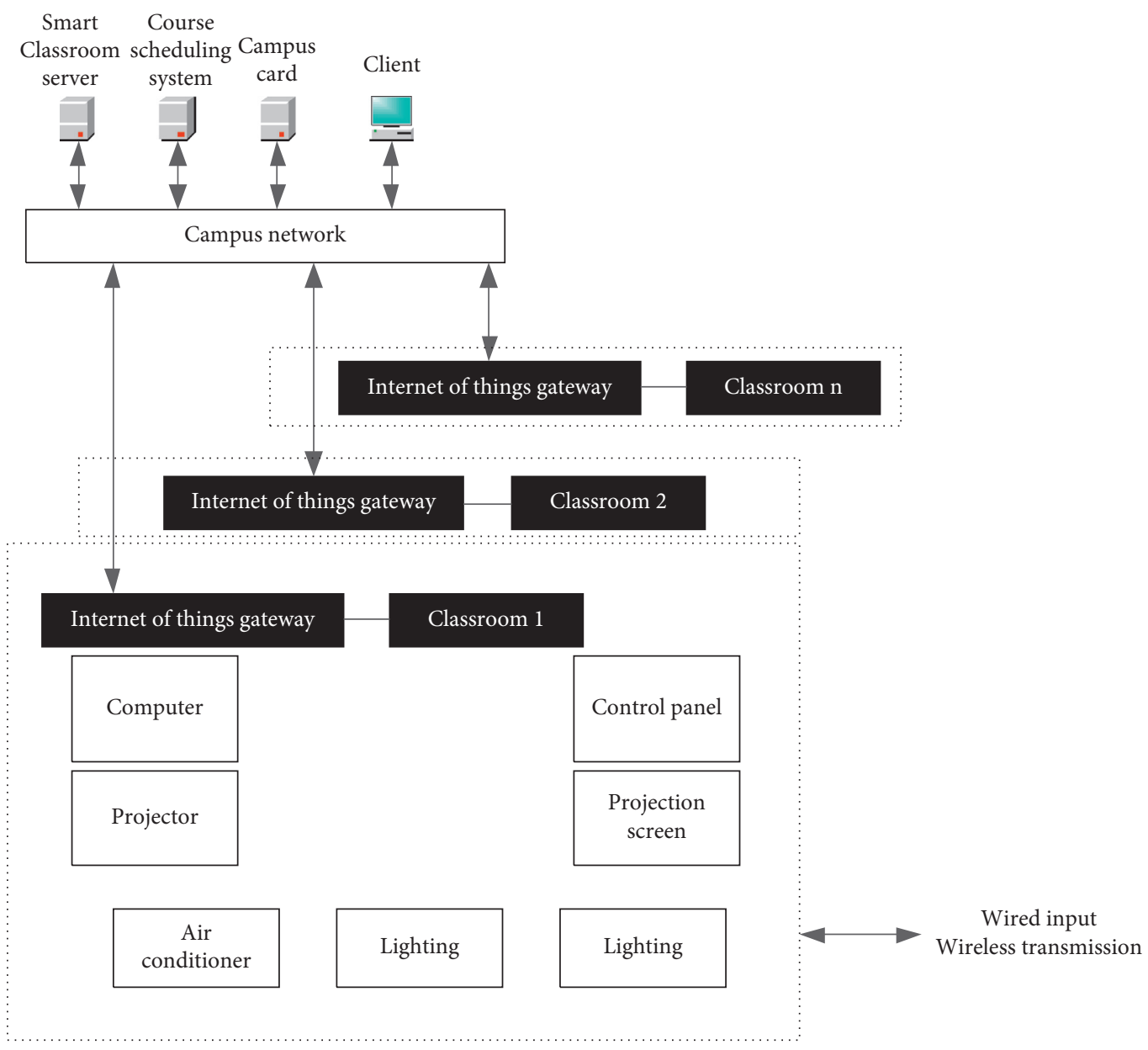

FIGURE 2: Smart classroom platform architecture.

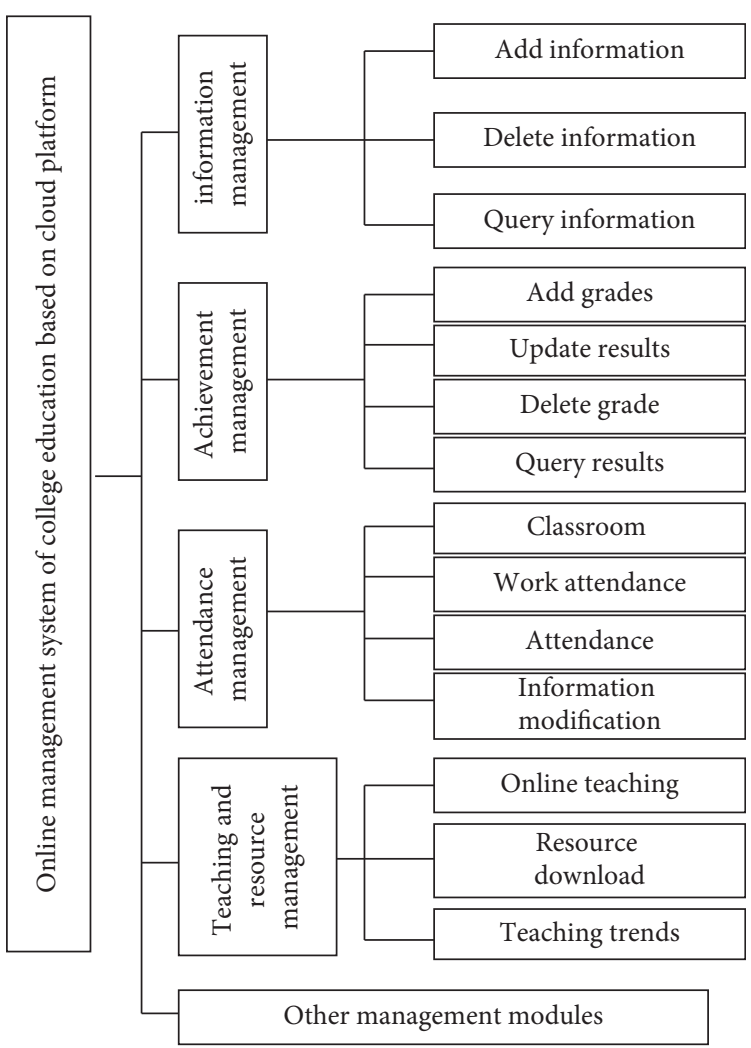

FIgURE 3: Functional structure of the platform. 


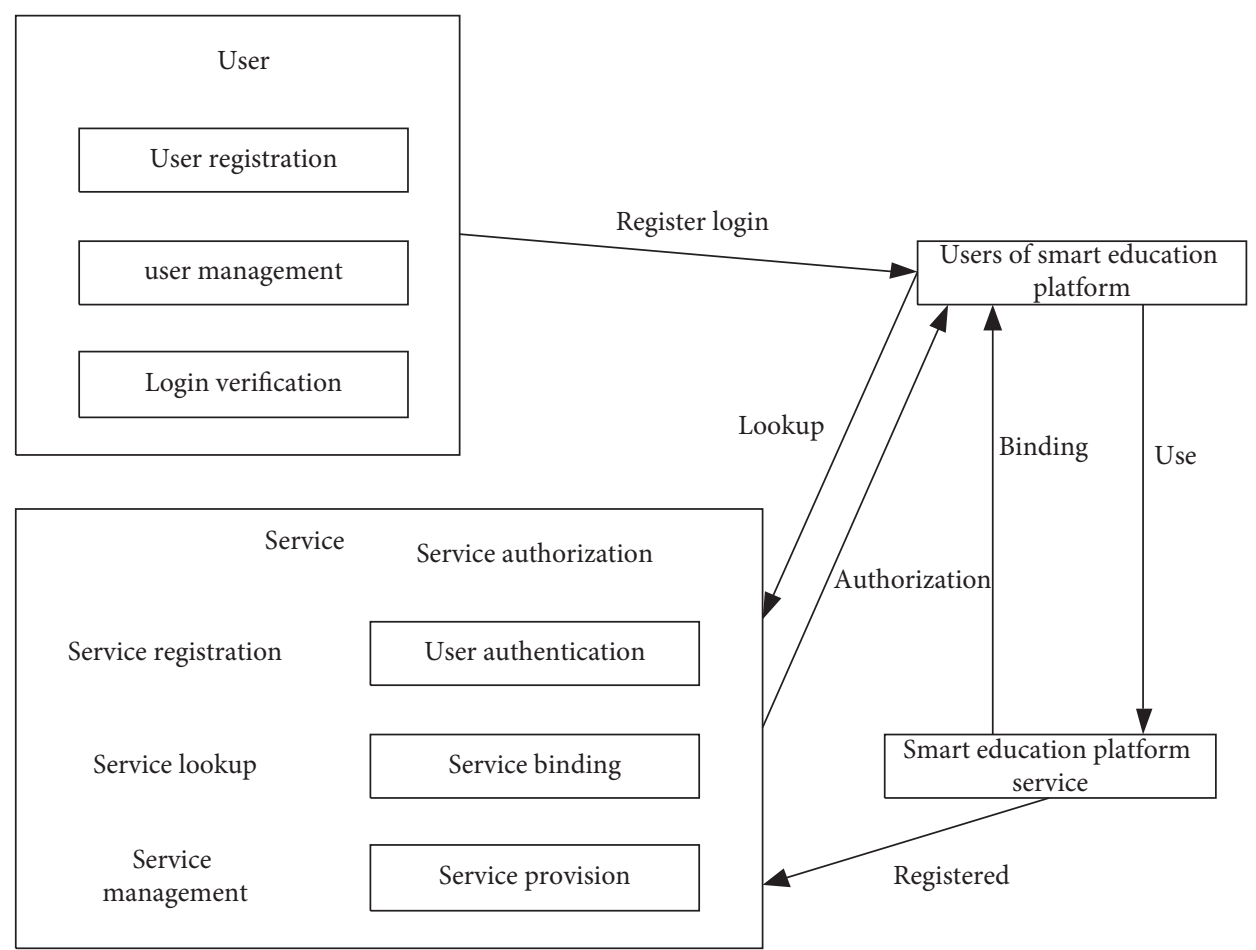

FIgURE 4: Smart education information cloud service layer structure.

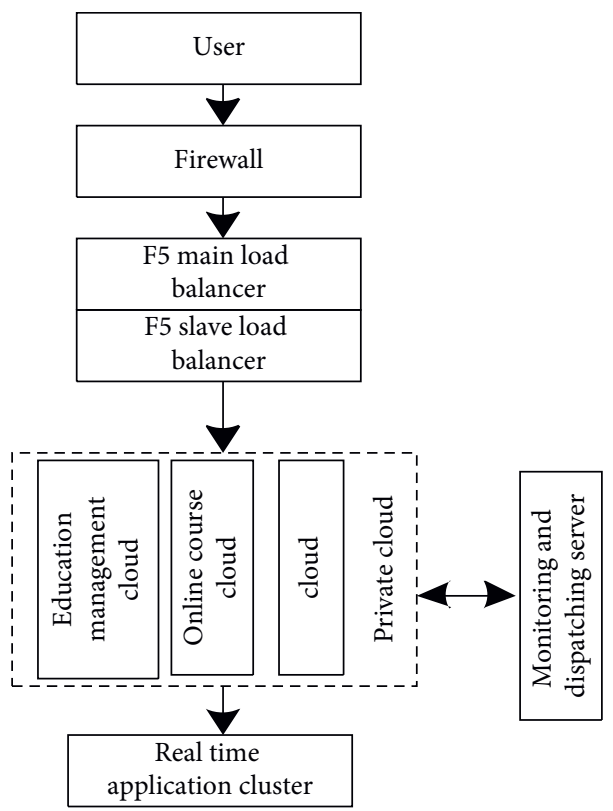

FIGURE 5: Deployment scheme of Internet of Things technology.

parameters to allocate resources to meet the access needs of highly concurrent users.

A monitoring and dispatching server mainly monitors the three following parts:

(1) Virtual machines. Red Hat's RHEV PowerShell API is used to monitor the running parameters of virtual machine, including memory, CPU, disk, and other running parameters.
(2) Physical servers. Nagios network, open-source software, is used to monitor and control the physical server, which can obtain the memory, $\mathrm{CPU}$, disk, and other running parameters of the physical server.

(3) Web applications. The MyARM software is used to monitor and control the response time of the Web application. 
During the deployment process of the Internet of Things technology, the relevant applications of online management of higher education are arranged within the virtual machines of the Internet of Things technology, and the deployed applications are cloned to templates; the Monitoring and Dispatching Server monitors the traffic of users on the platform, and the traffic of users on the platform is small, and the Internet of Things technology will open the virtual machines that are suitable for the resource parameters at this time; when the traffic of users on the platform increases, the Internet of Things technology searches for the physical servers with good operating conditions and uses the cloned templates to establish new virtual machines to meet the access needs of the upgraded users; when the traffic of users on the Monitoring and Dispatching Server monitoring the traffic of users on the platform significantly decreases, the Internet of Things technology will close the virtual organs to avoid the waste of cloud resources.

2.7.2. Implementation of Online Learning Module. Online learning module is the core of intelligent education information cloud service layer of intelligent education platform. Regardless of whether the online learning module has comprehensive teaching function, meticulous audit function and user experience will affect the online communication status of teachers, administrators, and students of intelligent education platform. The online learning module includes students, teachers, and administrators. The functional structure of the online learning module is shown in Figure 6.

In the online learning module, the administrator mainly examines the teacher to prepare lessons material and the student study resources; the curriculum examination guarantees the wisdom education platform teaching quality. Administrators provide data support for big data analysis courses by tracking students' learning progress and adopting continuous behavior tracking and data recording for the learning process.

The teacher completes the teaching preparation in the online learning module of the wisdom education platform and teaches the students by live broadcasting or videotaping. Students can learn the details of the course and choose and confirm it in the online learning module. Students can also learn both live and recorded courses. Students are required to complete practical training after the course ends. Otherwise, they cannot enter the next stage of the course.

\section{Platform Performance Test}

University students of grade 2020 are selected as the platform objects, and the total number of students of grade 2020 is 4855 . Using 15 computers as the node of IoT technology, including 1 master node and 14 computing nodes, the platform is built with Java language. A communication engineering major student is randomly selected as the test object of the platform. The running result of the platform to student management function is shown in Table 1.
A teacher majoring in communication engineering is randomly selected as the platform test object. The operation results of the management function of the platform for college teachers are shown in Table 2.

The university education manager is randomly selected as the platform test object. The operation results of the management function of the platform for university education managers are shown in Table 3.

The platform administrator is selected as the test object. The operation results of the management function of the platform administrator in this paper are shown in Table 4.

The platform test results in Tables 1-4 show that the test results of various management functions of the platform for students, teachers, education managers, and platform administrators are successful, and the response time of the platform for different test indicators of different users is less than $150 \mathrm{~ms}$. The platform test results show that the platform can meet the different needs of different users, and the platform response speed is fast, with high management performance.

This platform is used to allocate examination rooms and invigilator teachers to 2020 professional classes of a university. The allocation results of platform examination rooms corresponding to different professional classes are shown in Figure 7.

As can be seen from the test results of the platform in Figure 7, using this platform can reasonably arrange the examination room and invigilator teachers according to the examination needs of classes of different majors, which meets the examination management needs of colleges and universities. It is verified again that this platform has high management performance.

Throughput refers to the number of tasks processed by the platform per unit time. The throughput of the platform in this paper when the number of concurrent visits is 1000 is counted. The platform in this paper is compared with the new generation smart classroom architecture platform proposed in [4] and the smart education platform based on big data analysis technology proposed in [6]. The comparison results are shown in Figure 8.

As can be seen from the comparison results of the platforms in Figure 8, the platform in this paper has high throughput at different numbers of concurrent users. The throughput of the platform in this paper is significantly better than the new generation smart classroom architecture platform proposed in [4] and the smart education platform based on big data analysis technology proposed in [6]. In this paper, the platform uses the Internet of Things technology to establish the online management platform of college education. Using the parallel algorithm mode, one task can be divided into multiple tasks to run at the same time, so as to improve the data mining performance and computing efficiency. The Internet of Things technology can make the server realize load balancing; the platform cluster has good performance and effectively improves the throughput of the platform.

The average delay of intelligent education platform is the sum of data queuing delay and transmission delay. The average delay is used to evaluate the three power 


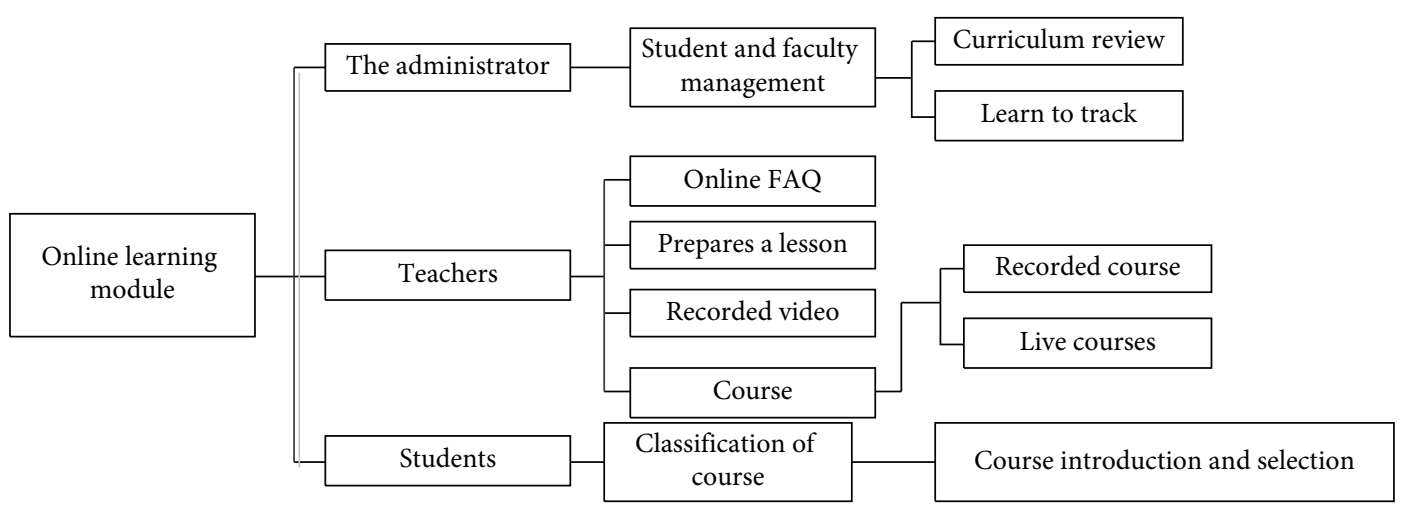

FIgURE 6: Structure of online learning module.

TABLE 1: Operation results of student management function.

\begin{tabular}{lcccc}
\hline Test index & Test object & Testing procedure & Test result & Response time/ms \\
\hline User login & Student & Log in after entering the account and password & Success & 5 \\
Information saving & Student & Enter the information and click save & Success & 4 \\
Modify user information & Student & Enter the modification information and click save & Success & 9 \\
Query course scores & Student & Click the required to query the course score and submit it & Success & 8 \\
Select course & Student & Click on the required to select a course and submit & Success \\
Delete course & Student & Click on the required to delete the course and submit & Success & 9 \\
Attendance query & Student & Click attendance query and submit & Success \\
Submit job & Student & Upload the job and click submit & Success \\
Online question & Student & Enter questions to be asked and submit & Success \\
Online test & Student & Click online test and submit & Success \\
\hline
\end{tabular}

TABLE 2: Operation results of teacher management function.

\begin{tabular}{lcccc}
\hline Test index & Test object & Testing procedure & Test result & Response time/ms \\
\hline User login & Teacher & Log in after entering the account and password & Success & 15 \\
Modify user information & Teacher & Enter the modification information and click save & Success & 11 \\
Query student list & Teacher & Click the required to query the student class and submit it & Success & 8 \\
Teaching plan release & Teacher & Upload the teaching plan and submit it & Success & 9 \\
Marking homework & Teacher & Submit the homework for review & Success & 7 \\
Enter results & Teacher & Upload student scores and submit & Success \\
Modify grades & Teacher & Click the student grade to modify and submit & Success & 11 \\
Course time release & Teacher & Enter course time and submit & Success & 9 \\
Query student scores & Teacher & Click the student achievement to be queried and submit it & Success \\
Query question bank & Teacher & Click the question bank to be queried and submit & Success & 6 \\
Answer questions & Teacher & Enter the answer to the question and save it & Success & 8 \\
\hline
\end{tabular}

TABLE 3: Operation results of education manager management function.

\begin{tabular}{|c|c|c|c|c|}
\hline Test index & Test object & Testing procedure & Test result & $\begin{array}{c}\text { Response } \\
\text { time } / \mathrm{ms}\end{array}$ \\
\hline Course number modification & $\begin{array}{l}\text { Education } \\
\text { manager }\end{array}$ & Enter the revised course number and submit & Success & 8 \\
\hline Arrange classes for students & $\begin{array}{c}\text { Education } \\
\text { manager }\end{array}$ & Enter class course and submit & Success & 9 \\
\hline Schedule classes for teachers & $\begin{array}{l}\text { Education } \\
\text { manager }\end{array}$ & Enter teacher course and submit & Success & 8 \\
\hline Classroom arrangement & $\begin{array}{c}\text { Education } \\
\text { manager }\end{array}$ & Enter the classroom number and submit & Success & 10 \\
\hline Examination room arrangement & $\begin{array}{l}\text { Education } \\
\text { manager }\end{array}$ & Enter the appropriate examination room and submit it & Success & 15 \\
\hline Query file & $\begin{array}{c}\text { Education } \\
\text { manager }\end{array}$ & Click the file to be queried & Success & 10 \\
\hline
\end{tabular}


TABle 3: Continued.

\begin{tabular}{|c|c|c|c|c|}
\hline Test index & Test object & Testing procedure & Test result & $\begin{array}{c}\text { Response } \\
\text { time/ms }\end{array}$ \\
\hline Modify financial information & $\begin{array}{c}\text { Education } \\
\text { manager }\end{array}$ & Enter financial information and submit & Success & 14 \\
\hline $\begin{array}{l}\text { Modification of educational } \\
\text { facilities }\end{array}$ & $\begin{array}{l}\text { Education } \\
\text { manager }\end{array}$ & Enter facilities to be educated and submit & Success & 14 \\
\hline Query employment information & $\begin{array}{l}\text { Education } \\
\text { manager }\end{array}$ & Click the employment information to be queried & Success & 7 \\
\hline Query personnel information & $\begin{array}{l}\text { Education } \\
\text { manager }\end{array}$ & Click the personnel information to be queried & Success & 8 \\
\hline
\end{tabular}

TABLE 4: Operation results of platform administrator management function.

\begin{tabular}{llccc}
\hline Test index & \multicolumn{1}{c}{ Test object } & Testing procedure & Test result & Response time/ms \\
\hline Login monitoring & Platform administrator & Implementation view user login & Success & 18 \\
Statistics of login times & Platform administrator & Click total login times and submit & Success & 14 \\
Online personnel statistics & Platform administrator & Click online person and submit & Success & 12 \\
Add teacher information & Platform administrator & Add teacher information and submit & Success & 18 \\
Delete teacher information & Platform administrator & Delete teacher information and submit & Success & 11 \\
Modify teacher information & Platform administrator & Modify teacher information and submit & Success & 13 \\
Add student information & Platform administrator & Add student information and submit & Success \\
Delete student information & Platform administrator & Delete student information and submit & Success & 9 \\
Modify student information & Platform administrator & Modify student information and submit & Success & 9 \\
View suggestions & Platform administrator & Click suggestions to view and submit & Success & 10 \\
\hline
\end{tabular}

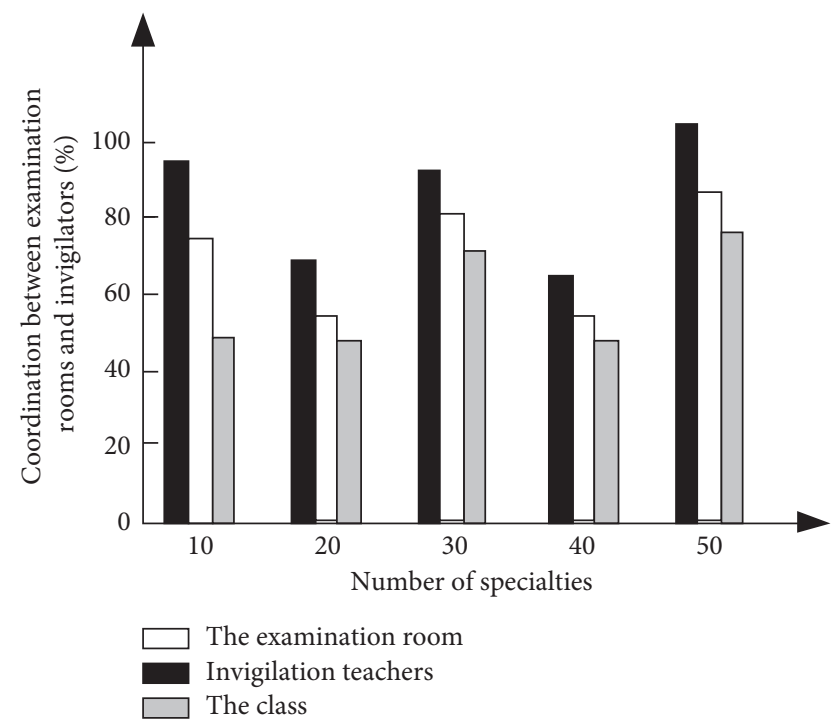

FIGURE 7: Allocation results of examination room and invigilator.

consumption monitoring platforms and compare the monitoring effects of different platforms. The results are shown in Figure 9.

According to Figure 9, although the delay time of the proposed platform increases, it is relatively stable, while the time delay of the other two platforms increases sharply with the increase of load. This is because the proposed platform preprocesses the power data before the user's power consumption, which reduces the noise, so as to ensure the clarity

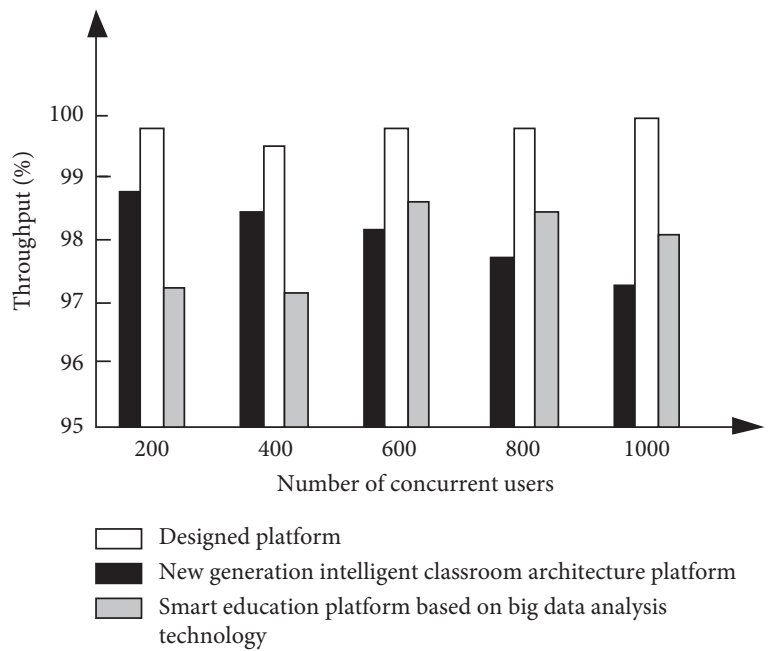

FIgURE 8: Platform throughput comparison.

of data, improve the monitoring accuracy, and speed up the monitoring time. The average monitoring delay is reduced.

The generalization ability of the proposed platform is verified by 500 iterative experiments, and the results are shown in Table 5.

As can be seen from Table 5, the F1 score of the proposed platform is within the interval [0.912, 0.968], and the AUC score is within the interval [0.893, 0.999]. Therefore, the F1 score and the AUC score of the proposed platform are both in a higher state, and the five groups of experiments show that the performance of the proposed platform is stable and 


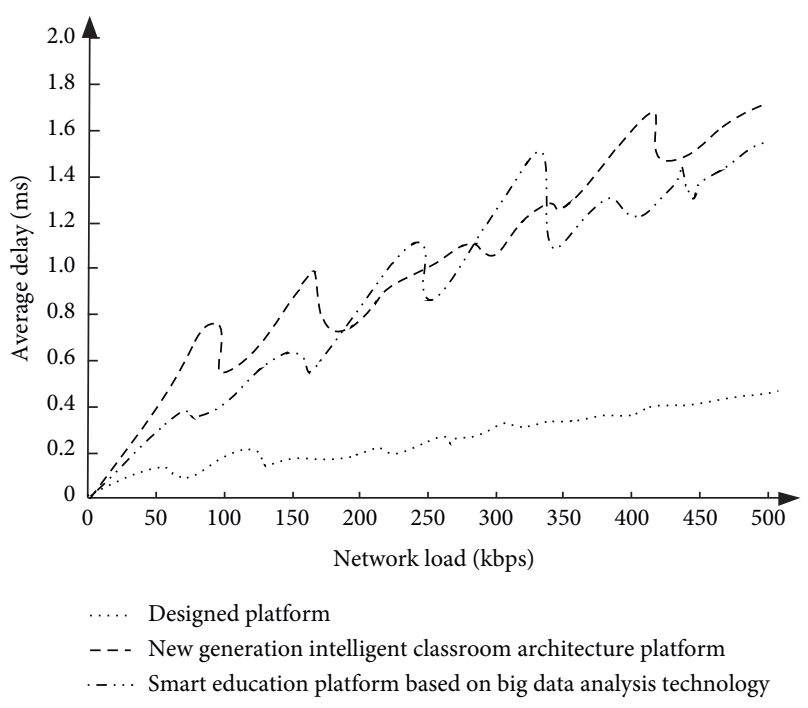

Figure 9: Average delay of different intelligent education platforms.

TABLE 5: Generalization capability of the proposed platform.

\begin{tabular}{lcc}
\hline Iterations/times & F1 fraction & AUC fraction \\
\hline 100 & 0.935 & 0.893 \\
200 & 0.952 & 0.993 \\
300 & 0.968 & 0.972 \\
400 & 0.946 & 0.990 \\
500 & 0.912 & 0.999 \\
\hline
\end{tabular}

there is no obvious difference. It is found that the F1 score fluctuates more than the AUC score according to the distribution of the two samples of data, because the F1 score is the judgment threshold, which leads to the oversensitivity of the data. After the experiment, it is found that the proposed platform not only guarantees that the data will not be oversensitive but also guarantees that the AUC score is all over 0.9. This is because the proposed platform uses wavelet denoising method to denoise the power data and then monitors the use of power data, screens and analyzes the effective signals, improves the precision rate and accuracy rate, and thus improves the F1 and AUC scores, thus strengthening the general performance of the proposed platform.

The PR curve is an important index in the process of monitoring abnormal power consumption in the intelligence classroom of the intelligence education platform. It is the ratio between the precision rate and the recall rate of abnormal power consumption.

As can be seen from Figure 10, the proposed platform has great advantages in the process of monitoring unconventional voltage, and the performance of the other two platforms is poor. Especially for the platform in [5], the PR curve of the proposed platform is smooth, and the proposed platform can even directly "wrap up" the PR curves of the other two platforms, which shows that the proposed platform has strong learning ability and thus proves that the proposed platform has more advantages

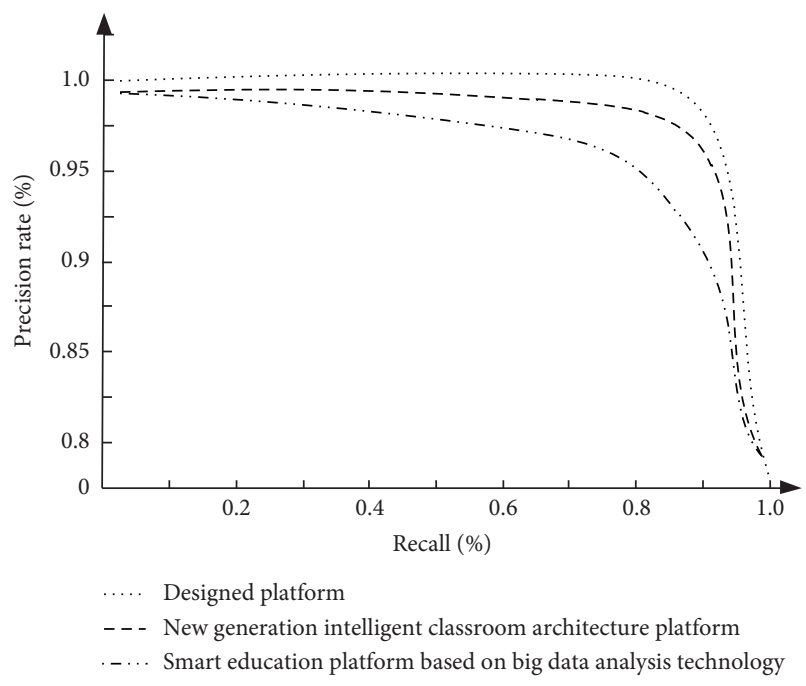

FIgURE 10: Abnormal power consumption PR curve of different platforms.

in the process of monitoring abnormal power consumption, because the proposed platform can normalize the power data in the process of monitoring the unconventional low-voltage power consumption, reduce the monitoring types, reduce the monitoring difficulty, improve the accuracy rate, and then enhance the PR curve.

\section{Conclusion}

This research has designed the university intelligence education platform based on the Internet of Things technology. The platform realizes the functions of examination result inquiry, online teaching, and attendance management. The intelligent classroom architecture based on Internet of Things technology is designed, and the intelligent classroom instruction is transmitted to the environment equipment controller of the designated classroom. Based on this, we design the function module and the cloud service layer of intelligent education information, deploy the IOT technology, and complete the design of intelligent education platform based on IoT technology. The platform test results show that the classroom assignment result of the platform is better, the throughput is higher, and the application delay is lower. The experimental results show that the platform has good application effect and reliability.

In the future, the proposed study is planned to be tested and used by adopting the deep learning based predictive models exploiting the IoT-based data.

\section{Data Availability}

The data used to support the findings of this study are available from the corresponding author upon request.

\section{Conflicts of Interest}

The authors declare that there are no conflicts of interest. 


\section{Acknowledgments}

This work was supported by General Research Project of Philosophy and Social Sciences in Jiangsu Universities in 2021 (2021SJA2124) and Science and Technology Project of Jiangsu Vocational College of Agriculture and Forestry in 2021 (2021kj58).

\section{References}

[1] M. D. Abdulrahaman, N. Faruk, A. A. Oloyede et al., "Multimedia tools in the teaching and learning processes: a systematic review," Heliyon, vol. 6, no. 11, Article ID e05, 2020.

[2] S. K. Mydhili, S. Periyanayagi, S. Baskar, P. M. Shakeel, and P. R. Hariharan, "Machine learning based multi scale parallel K-means++ clustering for cloud assisted internet of things," Peer-to-Peer Networking and Applications, vol. 13, no. 2, pp. 2023-2035, 2020.

[3] T. Yilmaz, R. Ozcan, I. S. Altingovde, and Ö. Ulusoy, "Improving educational web search for question-like queries through subject classification," Information Processing \& Management, vol. 56, no. 1, pp. 228-246, 2019.

[4] X. R. Wu, B. Q. Liu, and T. T. Yuan, "A new generation of smart class: concept, platform and system architecture," China Educational Technology, vol. 6, no. 3, pp. 81-88, 2019.

[5] Q. H. Zheng, B. Dong, B. Y. Qian et al., "The state of the art and future tendency of smart education," Journal of Computer Research and Development, vol. 56, no. 1, pp. 213-228, 2019.

[6] W. L. Shi and Y. H. Zhang, "Intelligent education platform design based on big data analysis technology," Modern Electronics Technique, vol. 43, no. 9, pp. 158-161, 2020.

[7] I. Heđi, I. Špeh, and A. Šarabok, "IoT network protocols comparison for the purpose of iot constrained networks," in Proceedings of the 2017 40th International Convention on Information and Communication Technology, Electronics and Microelectronics (MIPRO), pp. 501-505, IEEE, Opatija, Croatia, 2017 May.

[8] S. J. Andriole, "Social media analytics, wearable technology, and the internet-of-things," IT Professional, vol. 21, no. 5, pp. 11-15, 2019.

[9] G. Bodur, S. Gumus, and N. G. Gursoy, "Perceptions of Turkish health professional students toward the effects of the internet of things (IOT) technology in the future," Nurse Education Today, vol. 79, pp. 98-104, 2019.

[10] C. Engelhardt-Nowitzki, M. Aburaia, R. Otrebski, J. Rauer, and H. Orsolits, "Research-based teaching in digital manufacturing and robotics - the digital factory at the UAS technikum wien as a case example," Procedia Manufacturing, vol. 45, pp. 164-170, 2020.

[11] V. Mkrttchian, L. Gamidullaeva, A. Finogeev, and S. Chernyshenko, "Big data and internet of things (IoT) technologies' influence on higher education: current state and future prospects," International Journal of Web-Based Learning and Teaching Technologies, vol. 16, 2021.

[12] B. Monea, "Looking at screens: examining human-computer interaction and communicative breakdown in an educational online writing community," Computers and Composition, vol. 58, no. 2, Article ID 102605, 2020.

[13] J.-M. Liang, W.-C. Su, Y.-L. Chen, S.-L. Wu, and J.-J. Chen, "Smart interactive education system based on wearable devices," Sensors, vol. 19, no. 15, p. 3260, 2019.

[14] H. Tanveer, T. Balz, N. S. Sumari, R.-u. Shan, and H. Tanweer, "Pattern analysis of substandard and inadequate distribution of educational resources in urban-rural areas of Abbottabad, Pakistan," Geojournal, vol. 85, no. 5, pp. 1397-1409, 2020.

[15] T. Martin and R. Kimmons, "Faculty members' lived experiences with choosing open educational resources," Open Praxis, vol. 12, no. 1, p. 131, 2020.

[16] S. Pundir, M. Wazid, D. P. Singh, A. K. Das, J. J. P. C. Rodrigues, and Y. Park, "Intrusion detection protocols in wireless sensor networks integrated to internet of things deployment: survey and future challenges," IEEE Access, vol. 8, pp. 3343-3363, 2020.

[17] Y. M. Tukur, D. Thakker, and I. U. Awan, "Ethereum blockchain-based solution to insider threats on perception layer of iot systems," in Proceedings of the 2019 IEEE Global Conference on Internet of Things (GCIoT), IEEE.

[18] D. Satarupa, "Smart university utilising the concept of the internet of things(IoT)," Neurochemistry International, vol. 129, no. 10, p. 2022, 2019. 